MODELING, IDENTIFICATION AND CONTROL, 1986, VOL. 7, NO. 4, 163-171

doi: $10.4173 /$ mic.1986.4.1

\title{
Bridging the gap between aquaculture and the information sciences
}

\author{
J. G. BALCHEN $\dagger$
}

Keywords: Aquaculture, information sciences, modeling, control, instrumentation, optimization.

\begin{abstract}
The aquaculture industry is traditionally a low technology industry. But as demands on productivity, quality and availability are increasing the application of modern industrial technology is inevitable. After the fundamental problems of biology and of civil, mechanical and chemical engineering have been resolved, major progress may be expected through the application of the tools of the information sciences.
\end{abstract}

\section{Introduction}

Aquaculture, and its related industrial activity, has so far been mostly a low technology industry in the sense that the technical methods and apparatus employed are not particularly sophisticated. This is a rather reasonable situation since most aquaculture activities started out in the natural way with ordinary aquatic animals being fed and raised in captivity in some suitable way. From a rather elementary and perhaps primitive starting point, development of increasingly better methods have taken place resulting in many new possibilities which contain a lot of promise for future expansion.

Although there is a strong trend towards high technology in aquaculture, there is a need for 'the low technology' or even no-technology version of aquaculture in nations whose industrial development has not yet reached a high level. It is definitely possible to produce major amounts of food through aquaculture without using technical or industrial means at all. This mode of operation will be the most recommended method in many developing nations for a long time to come. In the industrialized nations, however, and particularly those with a high price of labour, high technology must be employed at an increasing rate. Such is the case in Norway where harvesting the ocean always has been important for the nation's survival and where the fishing industry has played an important role in the nation's economy for a long time. Since oil was discovered in Norway and the nation became more and more industrialized, relying more on high technology products, the fishing industry has experienced difficulties and is becoming more dependent upon state subsidies. Since Norway is extremely well suited for an aquaculture industry, there has been a strong growth in this field. In particular, the culture of salmonides has been successful. So far the economy of salmon farming has been very good in Norway.

Received 15 January 1987.

This paper was presented at the IFAC Symposium on Automation and Dataprocessing in Aquaculture, Trondheim, Norway, 18-20 August 1986.

This paper is reprinted with the permission of IFAC.

$\dagger$ Division of Engineering Cybernetics, The Norwegian Institute of Technology, 7034 Trondheim, NTH, Norway 


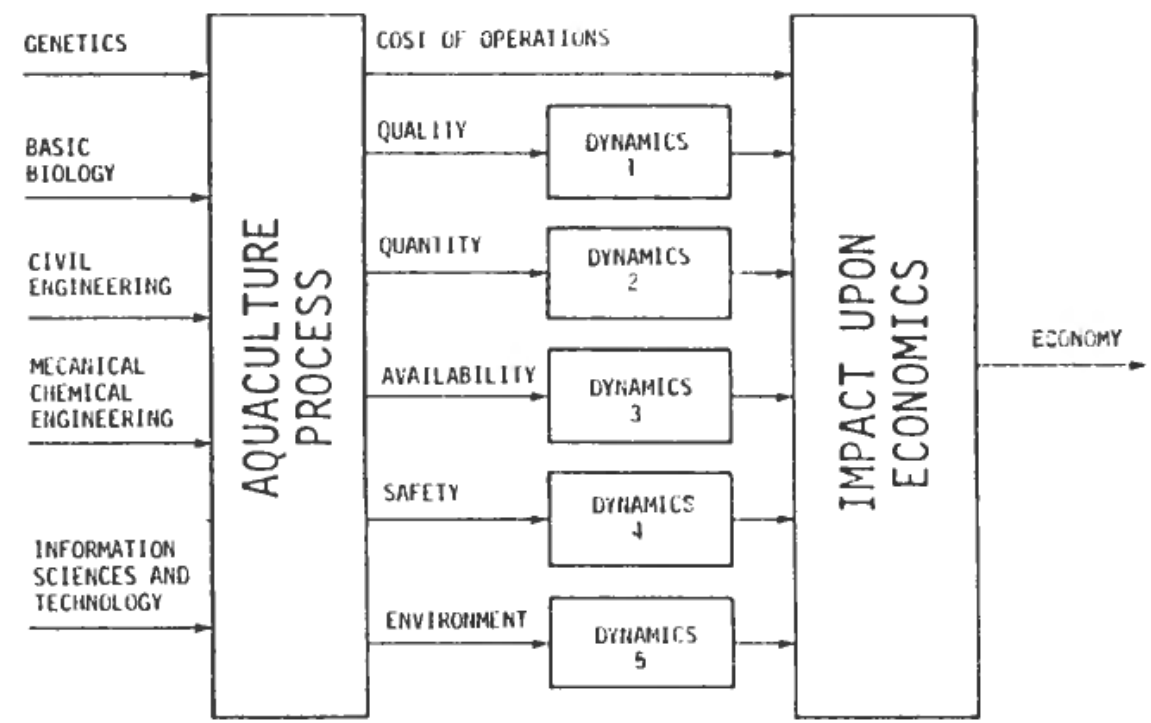

Figure 1. The impact of different techniques on the economy of an aquaculture process.

However, it is expected that the ever increasing cost of labour, energy, and other resources needed in aquaculture will lead to the development of more industrialized processes relying more on high-technology and less on human labour.

\section{The background for a high technology aquaculture}

As in most other activities encountered by man in the modern society, the search for better solutions is going on continuously and never seems to stop. In societies with free competition, quality, price and availability are the major driving forces leading to demands for improvements in the methods employed by the aquaculture industry. Aspects like safety, environmental impacts, restrictions imposed by laws, and so on, give even more pressure in the same direction.

Improvements in the aquaculture industry may be expected over a wide spectrum of fields resulting from research and development activities. Such fields may be in genetics, biotechnology, pharmacy, chemical engineering, civil engineering and the information sciences. Particular emphasis will be placed on the role of the information sciences in this paper.

Figure 1 attempts to illustrate how different techniques or methods employed in an aquaculture facility may influence the economics of the operation in different ways. First of all there is a direct influence caused by the cost of equipment and materials. This influence is usually rather direct and not associated with particular dynamic effects. Another connection with the techniques used is the quality of the product produced. The quality influences the price obtained for the product, but it is associated with a rather pronounced dynamic effect because it may take a long time to build up an image of high quality in the market and likewise the image may stay for some time even though the quality goes down.

The same type of phenomenon will apply to the relationship between the techniques employed and the quantity of products produced. The earnings will be more or less proportional to the quantity, but there is a pronounced dynamic effect in that quantity does not come immediately, but after may be a number of years, depending on the species under consideration.

Availability is a rather complex term which may include such factors as the product being present at the proper time; in the proper location, in a convenient 
form, etc. Only some of the techniques employed in the aquaculture process will influence availability, namely those related to the very latest parts of the production process such as packing, transportation and distribution. The dynamic effects of availability upon economics may be both short range and long range because of its influence upon the image of the product.

Safety in aquaculture operations relates to the personnel involved. Some aquaculture techniques are basically safe whereas others, particularly those involving open-water operations, may involve a certain hazard and inconvenience to the personnel. The economic effects of varying degrees of safety are not quite obvious, but it must be expected that in a competitive situation the facility with the best working conditions will attract the best people and thus become a leader in the long run.

Some aquaculture techniques have more negative environmental impacts than others, for instance in terms of pollution. The degree of environmental impact is mostly regulated by law and the economic consequences may be quite severe and long range if the given specifications are not met.

The conclusion to be drawn from the above is that the economics of an aquaculture operation may be critically dependent upon the techniques employed and that the dynamic effects may be pronounced. The information science-based techniques are generally characterized by a relatively direct positive impact upon economics in terms of improvements in quality, quantity, availability, safety and environmental conditions, whereas the cost of implementation usually is rather modest.

\section{The role of information sciences in aquaculture}

Those branches of the information sciences which will be considered in the present context are:

\section{Mathematical/numerical techniques in}

Modeling as applied to physiology, ecology, physical systems, behavior, economy, etc.

State and parameter estimation applied to models as above.

Optimization of plant design and operating conditions.

Population dynamics.

\section{Instrumentation and communication involving}

Primary measurements of biological and physical quantities such as temperature, oxygen, ammonia, fish size, fish number, etc.

Conversion of primary measurements to convenient format.

Transmission (analog or digital) to distributed or centralized data storage and retrieval.

Operator/process-communication facility including displays, recording and storage.

Remote communication to other facilities and executive offices.

\section{Control involving}

Manipulation of biological and physical conditions based upon measured or estimated (calculated) states of the plant.

Manipulation of animal behaviour. 
Manipulation of physiological states of animals based upon measured and estimated states.

\section{Data processing involving}

Computational facilities for numerical computations as above

Production planning.

Statistics.

Administrative routines.

Book-keeping.

Even though the science and industry of aquaculture has so far not been particularily active in employing principles and tools from the information sciences, there is no doubt that this is a field of extraordinarily high potential and growth. Since biological processes as encountered in aquaculture are generally complex, dynamic, multivariable and non-linear in nature, they require the best tools in terms of mathematical and computational methods in order to arrive at successful management through control and optimization. Some examples from actual and potential applications of systems and information sciences to aquaculture will illustrate this point.

\subsection{Modeling and optimization of growth}

The first stage of many aquaculture processes is that of producing larvae of aquatic animals from eggs carried by females. In this phase of the process there are a lot of problems which lend themselves to solutions aided by information technology. Such problems are numerical modeling of the maturation of the eggs towards hatching depending on temperature and initial state, the modeling of growth and population of larvae depending on temperature, food, density etc. Information gained from a numerical model of such a process together with measurements taken from the real process may be used in manipulating the process so as to arrive at a desired result. An example is hatching of the eggs at a desirable instant in time by manipulating the temperature and having the larvae available at the proper time. Also, manipulating the feed at the proper time and in proper quantities is an information problem.

When larvae or small fry are put into a bigger tank, a pond, a floating pen or some larger closed-off area like a bay or a fjord, it is important to monitor and control the biological and physical conditions of this environment. Numerical modeling of hydrodynamic conditions, temperature, oxygen concentration, ammonia concentration, bacteria and virus concentration as well as updating of such calculated variables against measurements taken from the real system is referred to as state estimation. It makes it possible for the operator to have an overview of the conditions in his plant at any time and to introduce proper actions in order to change or improve these conditions. Such an action could be to increase aeration to improve oxygen concentration. In systems with chemical or biological treatment of the water and recycling of used water to economize with water and energy, modeling and control of process conditions (state variables) becomes a necessity.

In aquaculture the growth of the animal is one of the most important factors for the success of the enterprise. More generally the physiological state of the animal 
describing the development of proteins, lipids, bone structure, pathological phenomena, etc., are decisive for the success of the process and should be modeled and monitored towards a given goal of quality.

The population dynamics of individuals in an aquaculture facility also has strong relations to the physiological state and the environmental conditions and is governed by natural death, disease and predation. Together, models of animal physiology (describing growth and quality) and population dynamics form the bases for estimating (monitoring and controling) the proper functioning of the aquaculture facility.

Optimization of growth and timing of harvest from an economic point of view is one of the most important aspects of the management of an aquacultural facility. In this context a model of growth and population as mentioned above is necessary for the derivation of optimal strategies. Much attention has been paid to this problem and it is a good example of the interaction between systems and information sciences on the one side and aquaculture science on the other.

\subsection{Instrumentation}

The collection of primary data from sensors located in a facility, the conversion of the information into convenient formats, the transmission of the information and its presentation, storage and retrieval is commonly referred to as instrumentation technology. This is one of the cornerstones of the information sciences. For all of the above-mentioned tasks, reliable and precise information from the facility is of high importance. New and sophisticated sensing systems and new techniques for data transmission such as multichannel digital transmission and fibre optics are finding their way into aquaculture facilities with great success. Modern computer-driven graphical displays are very convenient for the plant operator to get information displayed to him from any point in the plant at any time. Furthermore he can have trends of different variables recorded with the possible early discovery of abnormal situations.

\subsection{Control}

Control of an aquaculture facility can take very many different forms. An elementary and obvious control task is to manipulate the energy supply to some volume of water to control its temperature. Another is to manipulate the flow and composition of feed to achieve optimal growth according to model predictions. An even more complex control objective is to influence the movement and location of the population of fish in a large enclosed area by means of strong electrical fields (repulsion) and by means of conditioning using sound signals combined with food (attraction).

\subsection{Data processing}

Data processing has many applications in aquaculture operations, in addition to those mentioned above, in which computers play an important role. Management of large amounts of data which may give statistical information useful for the planning and operation of the plant is such an example. Furthermore, accounting, records of inventory, production planning and programmed preventive maintenance are functions well suited for computers. 


\section{The transfer of knowledge across barriers between specialist fields}

One often hears aquaculturists say that 'there is no need for complicated instruments and control in aquaculture' or 'the aquaculture processes are too complicated to be described by mathematics' or 'the economics of an aquaculture facility does not permit the use of highly complex technology'.

Such statements may sometimes be correct, but most often are they caused by lack of insight. Many aquaculturists are trained and have experience in operations that are non-industrial in their mode of operation. They are used to the manual operation, that is, turning on the heat when the water is too cold and turning it off when it is too warm, starting a pump when the water level is too low and so on. Furthermore, they are accustomed to observing the conditions in their facility with their human senses rather than measuring by technical instruments. There seems to be a negative attitude among some towards technology caused either by experience with bad equipment or most probably a prejudice created by cultural background. Obviously, bad equipment does not function well, but well-designed equipment most often functions so well that the operator later on cannot imagine how he could do without it. Technical equipment is usually not expensive compared to its performance in terms of economic gains in quality and quantity of products. When the demands on volume and quality in aquaculture increase, there is really no alternative to large-scale application of technology. In this evolution the information technologies naturally will come in after the civil-engineering, mechanical engineering, and chemical engineering problems have been resolved.

There is an apparent need to break down some of the existing barriers preventing successful application of the proper technologies in aquaculture. The best means for doing that is the efficient transfer of knowledge from one field to the other. This again leads to the need for education. Systematic education in aquacultural engineering and particularly in the methods of information technologies in aquaculture is practically non-existent even in the most developed countries. Some countries are in the starting phase, but the number of candidates produced is still too small.

In addition to a more efficient education, there is an urgent need for research and development programmes in which information scientists, biologists and other specialists cooperate in both formulating and solving problems. So far, this has been the case in only a few programmes.

\section{Perspectives in relations between aquaculture and information sciences}

There are some very exciting perspectives for the future development of aquaculture in which the information sciences play a significant role. A few such perspectives are outlined below.

One field of great potential is that of computer-aided design of numerical models for different biological processes encountered in aquaculture based upon elementary knowledge from biochemistry, physics and so on, and observations from the real process. Examples of such processes are

Growth and distribution of phytoplankton and zooplankton in aquaculture water masses.

Growth and distribution of bacteria and virus in water masses and animals.

Growth of animals. 
Computer-aided design of numerical models is a technique in which a computer is given all available knowledge about the actual process in terms of basic relationships and observational data and then determines the model with its parameters which best fits the given information. The tool in this case is a computer with graphics output which makes it possible for the operator to interact at any time with his own knowledge and to evaluate the results. The application of computergenerated numerical models of aquacultural processes is primarily for the purposes of estimation, control and optimization. 'Early warning '-systems which predict future occurrences of undesirable conditions is an example of an estimation application. The economic value of such predictions leading to proper preventive actions may be highly significant in that major losses may be avoided.

The new trends in computer-aided instrumentation and data transmission comprise a number of very promising techniques applicable to aquaculture. Such is the case for instance in fibre optics for the transmission of large amounts of data at a rapid rate from remote locations in the aquaculture facility to a central control room. A fibre optical transmission is characterized by its insensitivity to electrical disturbances such as lightning and to the influence of water and corrosion.

Another field of great potential in aquaculture is image processing which can be applied to the estimation of animal populations and animal size, the retrieval of data from remote sensing of environmental parameters and the monitoring of animal behaviour. All these applications are characterized by an immense amount of data which does not easily lend itself to collection by means of point measurements, but which is very typical in biological systems. The primary sensing devices such as television cameras and infrared detectors are now available in great variety at low prices. The requirement on computational capacity for the realization of image processing algorithms has until recently lead to the use of fairly large computers. However, this is now changing since specialized single-chip processors are now being developed and manufactured which definitely will make image processing both efficient and inexpensive and thus a very promising tool in the aquaculture industry.

Control of animal behaviour is not an important issue when the animals are confined to small areas like tanks, ponds or floating pens. However, if the animals are moving rather freely in larger areas, such as closed-off fjords, bays or sounds, the monitoring and manipulation (control) becomes a significant issue. Such is the case for instance in the proposed raising of salmon and lobsters in large closed-off areas in Norway. In the case of salmon, two significant control problems occur, namely that of preventing the salmon from going out of the area into the ocean and that of collecting the salmon at given times for feeding, counting, weighing, medication, and harvesting. Because mechanical devices for fencing off an area have proven to be impractical due to the collection of debris, much work has been done in developing electrical and other types of non-mechanical devices which stop the fish, but let the water and debris through. These techniques are promising for very large-scale fish farming in semi-natural locations.

The manipulation of fish behaviour with the aim of collection (attraction) can be done using conditioning. The fish is then 'trained' to react to combination of two signals for instance one being food and another being either sound or light (or maybe both). Experience shows that it is very easy to manipulate fish behaviour in this way and make systems for the collection of large amounts of fish over great distances. This may lead to the development of fish farming facilities with high 


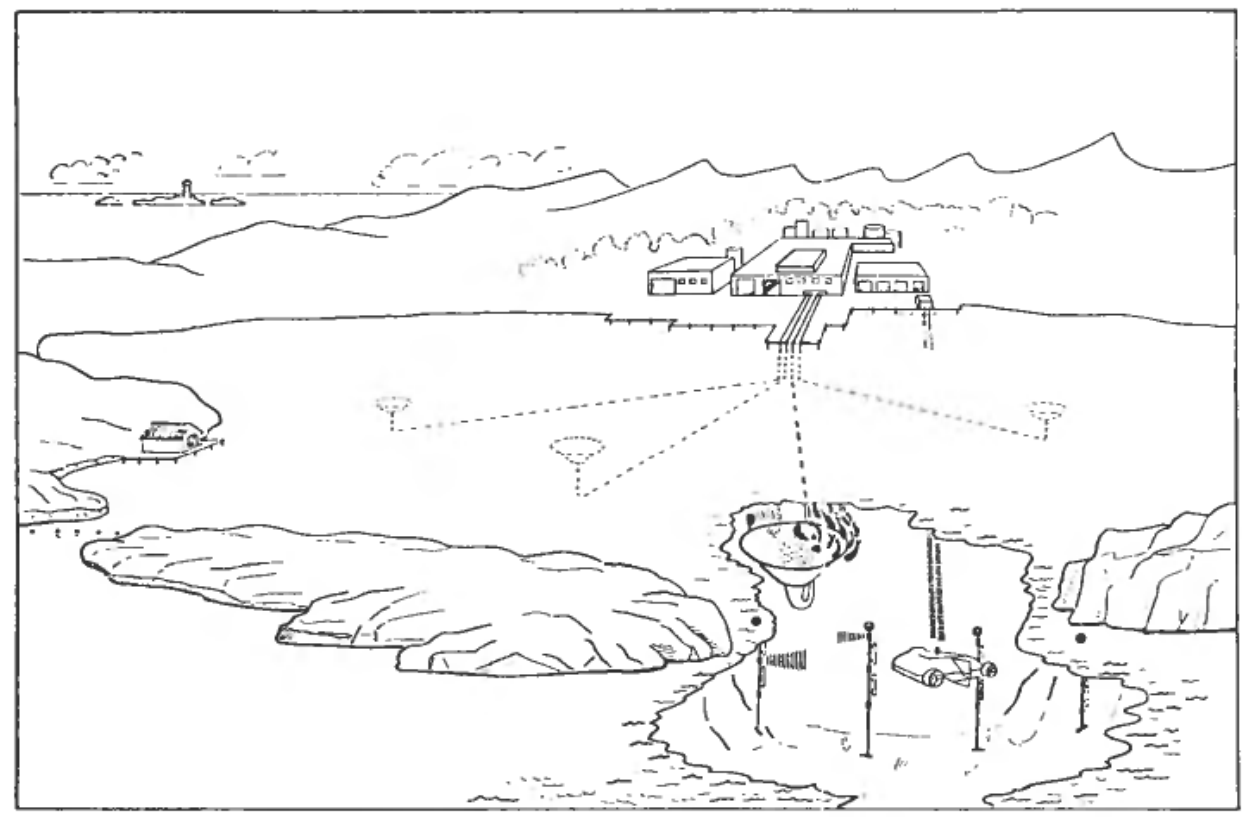

Figure 2. Proposed facility for fish farming in large fenced-off coastal areas.

capacity and very high productivity.

Figure 2 indicates how a facility for production of salmon in a fenced-off natural area on the coast of Norway is supposed to work. Such facilities have been under development for a number of years. The two inlets shown in Fig. 2 are equipped with electrical fences consisting of vertical electrodes between which strong electrical current pulses are passed. These pulses create electrical potentials in the water to which the fish are very sensitive and react by repulsion. However, it is difficult for the fish to determine the direction of the electrical field so as to choose the proper direction of escape. It has been discovered that by installing light sources which flash synchronously with the electrical pulses, the fish receives the necessary directional information so that it avoids the electrical shocks. Depending on the distance between the electrodes and the strength of the electrical current pulses, the fish may be made to stay at a minimal distance of 5 to 7 metres.

In Fig. 2, four feeding stations are indicated employing conditioning with sound sources located at each unit. Before the food is relcased, a sequence of low frequency sound pulses is transmitted to which the fish has been conditioned. The fish then will swim towards its feeding station and wait for the food to be released after a short time. In the case shown, the food is pumped from a centralized facility onshore containing food processing and storage; product processing, storage and packing and centralized monitoring and control systems.

A facility such as that shown in Fig. 2 is expected to yield both very high productivity and product quality which are the basic requirements for good economy.

\section{Conclusion}

It has been pointed out that the application of the results and tools from the information sciences will be beneficial to the economy of the aquaculture industry 
particularly in industrialized countries. The economy of an industry is a direct measure of its productivity, quality of products and its ability to cope with influence upon the environment. Since the price of computing power and advanced instrumentation is decreasing very fast and the capacity and quality is increasing, the motivation for bridging the gap between aquaculture and the information sciences is further enhanced. There can be no doubt that the information sciences will play a significant role when aquaculture grows out of its infancy and becomes a large-scale, highly productive industry with high demands on quality, price and availability.

\section{GENERAL REFERENCES}

Balchen, J. G. (1979). Modeling, prediction, and control of fish behaviour. In C. T. Leondes (ed.) Control and Dynamic Systems (New York: Academic Press), pp. 99-146.

Balchen, J. G. (1981). Recent progress in the control of fish behaviour. Proc. IFAC 8th World Congress, Kyoto, Japan, 1981.

Botsford, L. W., Rauch, H. E., and Shleser, R. (1974). Application of optimization theory to the economics of aquaculture. Proc. World Mariculture Society, 5, 387-401.

Botsford, L. W., Van Olst, J. C., Carlberg, J. M., and Gossard, T. W. (1977). The use of mathematical modeling and simulation to evaluate aquaculture as a beneficial use of thermal effluent. Proc. 1977 Summer Computer Simulation Conference, pp. 405-410.

GardNer, M. B., and MCCAUley, J. (1982). Micro-computers in aquaculture: valuable management tools. Aquaculture Magazine, Sept./Oct., 22-26.

Hvang, N., WanG, J., and Fujimura, T. (1976). A model for estimating prawn populations in ponds. Aquaculture, 8, 57-70.

MCNown, W., and SeIreg, A. (1983). Computer optimum design and control of staged aquaculture systems. J. World Mariculture Soc., 14, 417-433.

Powers, J. E. (1973). Dynamics in a salmon culture pond. Simulation, 21, 69-72.

SANNOmiYa, N., and Matuda, K. (1984). A mathematical model of fish behaviour in a water tank. IEEE Trans. Systems, Man and Cybernetics, 14, 157-162. 